\title{
Fixed versus dynamic subaortic stenosis: Hemodynamics and resulting differences in Doppler echocardiography and aortic pressure contour
}

\author{
Joon Hwa Hong, MD, PhD, ${ }^{a}$ Hartzell V. Schaff, MD, ${ }^{a}$ and Rick A. Nishimura, MD, ${ }^{\mathrm{b}}$ Rochester, Minn
}

\footnotetext{
From the Divisions of ${ }^{\mathrm{a} C}$ ardiovascular Surgery, and the ${ }^{\mathrm{b}}$ Cardiovascular Diseases, Mayo Clinic, Rochester, Minn J.H.H. is currently with Department of Thoracic and Cardiovascular Surgery, Heart Institute, Chung-Ang University, Seoul, South Korea.

Disclosures: Authors have nothing to disclose with regard to commercial support.

Received for publication Sept 14, 2015; revisions received Oct 21, 2015; accepted for publication Oct 22, 2015; available ahead of print Nov 17, 2015

Address for reprints: Rick A. Nishimura, MD, Division of Cardiovascular Diseases, Mayo Clinic, 200 First St SW, Rochester, MN 55905 (E-mail: rnishimura@mayo.edu).

J Thorac Cardiovasc Surg 2016;151:883-4

$0022-5223 / \$ 36.00$

Copyright (C) 2016 by The American Association for Thoracic Surgery

http://dx.doi.org/10.1016/j.jtcvs.2015.10.082
}

It is sometimes difficult to distinguish a dynamic left ventricular outflow tract (LVOT) obstruction caused by hypertrophic cardiomyopathy from a fixed obstruction caused by discrete subaortic stenosis. This distinction is important, because both catheter-based and surgical interventions differ for these 2 conditions. Both diseases have presenting symptoms of a loud systolic murmur, hypertrophy of the left ventricle, and a high gradient across the LVOT in the absence of an abnormal aortic valve. The pathophysiology of the obstruction differs between these 2 entities, however, and can be identified by both Doppler echocardiography and direct pressure measurements. ${ }^{1,2}$ The aim of this report is to demonstrate the differences between fixed and dynamic subaortic stenosis by showing typical examples of direct pressure tracings and continuous-wave (CW) Doppler echocardiography images of both and correlating the differences with pathophysiologic findings.

\section{EXAMPLES}

\section{Fixed Subaortic Stenosis}

Images in Figure 1, $A$, show the pressure tracing and $\mathrm{CW}$ Doppler echocardiographic signal observed in fixed subaortic stenosis. The intraoperative direct pressure tracing curves demonstrate slowed aortic upstroke throughout systole. The CW Doppler image shows a velocity contour that peaks in early to mid systole; this is also observed in valvular aortic stenosis.

\section{Dynamic LVOT Obstruction}

Images in Figure 1, $B$, show the pressure tracing and CW Doppler echocardiographic signal observed in classic dynamic LVOT obstruction. In the intraoperative direct pressure tracing curves, the aortic upstroke at early systole has a rapid upstroke and then becomes flat. In addition, the

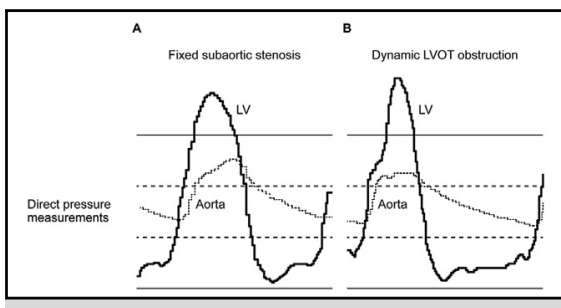

Pressure curves: fixed versus dynamic left ventricular outflow tract obstruction.

Central Message

CW Doppler and pressure contours of the aorta and left ventricle are important to distinguish fixed from dynamic subaortic obstruction.

See Editorial Commentary page 885

left ventricular pressure curve increases at end systole because the degree of obstruction is at a maximum at this time. The CW Doppler echocardiographic tracing shows slowly increasing velocity with a peak at systole-the so-called dagger shape of the velocity curve-which is the characteristic finding of dynamic LVOT obstruction.

\section{DISCUSSION}

In fixed subaortic obstruction, the obstruction starts during aortic valve opening and continues throughout systole. The aortic upstroke has a slow rate of increase, beginning at early systole and continuing throughout the rest of the ejection period. The left ventricular pressure contour has a symmetric shape with the peak in mid systole. ${ }^{2}$ The Doppler velocity through the obstruction then peaks in early to mid systole.

The unique mechanism of the increasing LVOT pressure gradient in hypertrophic cardiomyopathy is produced by systolic anterior motion (SAM) of mitral valve (MV) leaflets. During systole, as blood flow accelerates across the LVOT, MV leaflets are pushed into the LVOT, resulting in SAM of the MV and LVOT obstruction. In addition, because the LVOT obstruction mechanism is dynamic, the peak pressure gradient between the left ventricle and aorta in hypertrophic cardiomyopathy is delayed until late systole, when obstruction caused by SAM of the MV occurs. ${ }^{2}$ For this reason, the left ventricular pressure contour increases in late systole. This results in the gradient across the 

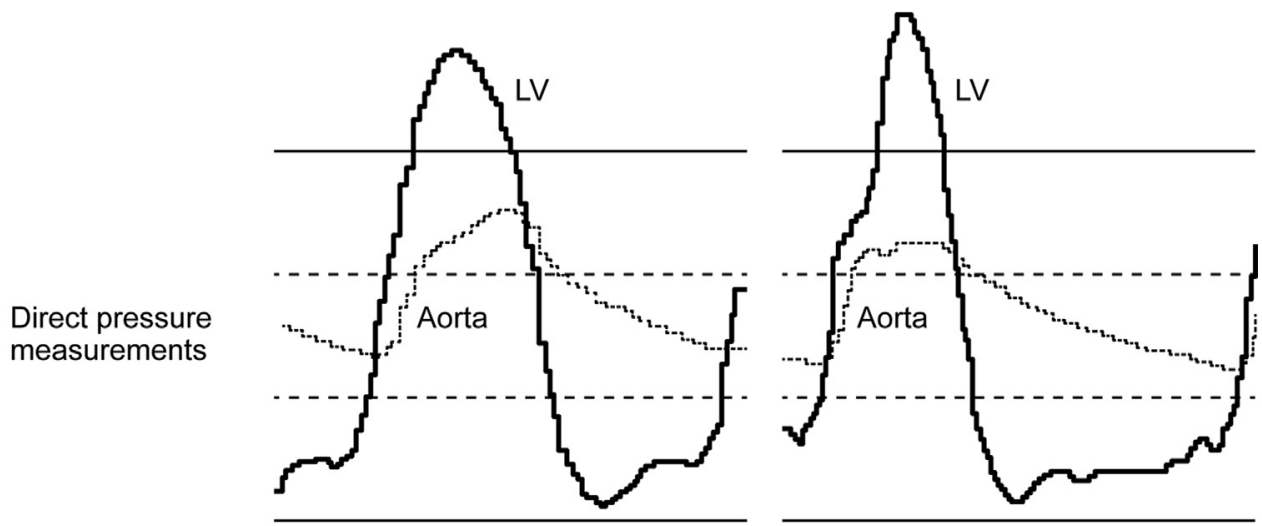

ECG
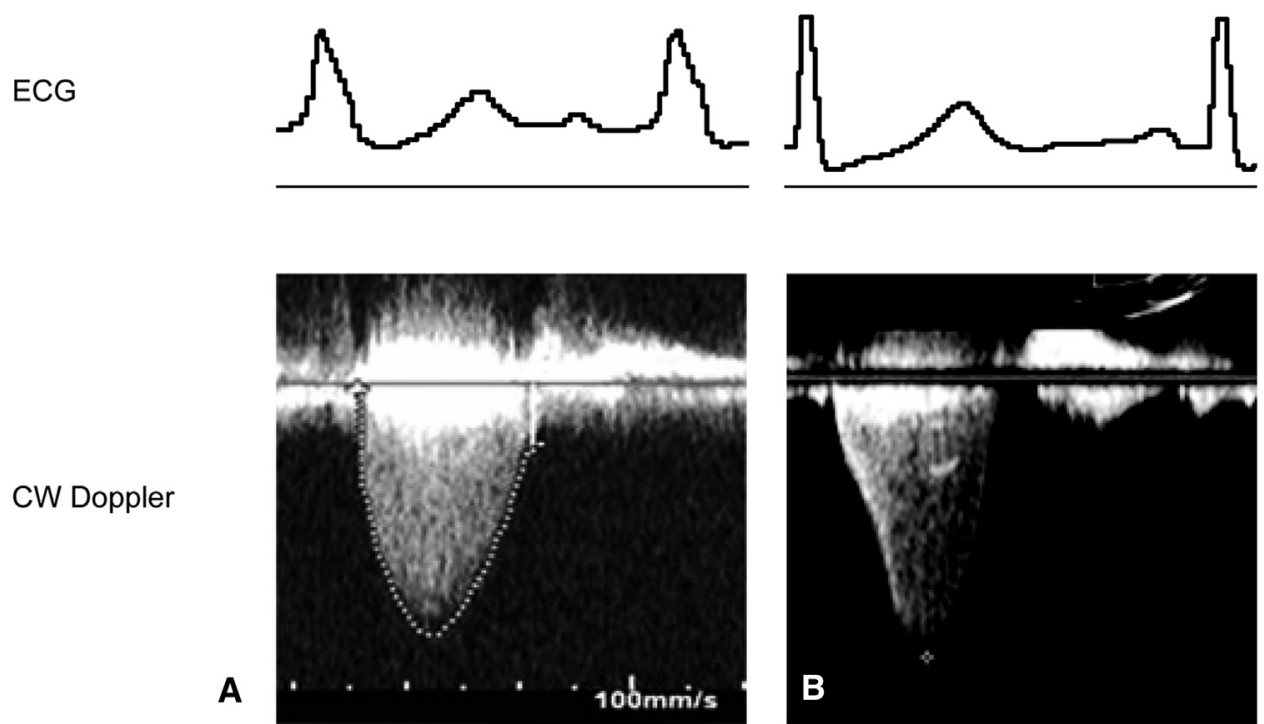

FIGURE 1. Examples of direct pressure measurements and continuous-wave $(\mathrm{CW})$ Doppler echocardiography images of fixed subaortic stenosis (A) and dynamic left ventricular outflow tract ( $L V O T)$ obstruction (B). The corresponding electrocardiography $(E C G)$ tracings are also shown. $L V$, Left ventricle.

LVOT peaking in late systole, which produces the dagger-shaped signal on CW Doppler echocardiography. ${ }^{3}$ In addition, because LVOT obstruction is minimal at early systole, when there is no SAM, the pressure generated by the left ventricle is transferred to the aorta quickly, establishing the quick aortic upstroke during early systole. The aortic pressure then rapidly decreases during mid and late systole, when significant obstruction by SAM of the MV limits pressure transfer from left ventricle to aorta. This forms the spike and dome pattern of the aortic pressure contour, as shown in Figure 1, $B$. This typical pattern becomes more prominent after premature contraction. Thus in patients with the typical findings of a fixed obstruction, a concomitant dynamic obstruction can be diagnosed if there is a spike and dome pattern on the aortic pressure trace on the beat after a premature contraction.

In conclusion, features of the CW Doppler echocardiographic signal and pressure contours of the aorta and left ventricle provide important information to distinguish between fixed and dynamic subaortic obstruction.

\section{References}

1. Geske JB, Cullen MW, Sorajja P, Ommen SR, Nishimura RA. Assessment of left ventricular outflow gradient: hypertrophic cardiomyopathy versus aortic valvular stenosis. JACC Cardiovasc Interv. 2012;5:675-81.

2. Nishimura RA, Carabello BA. Hemodynamics in the cardiac catheterization laboratory of the 21st century. Circulation. 2012;125:2138-50.

3. Nishimura RA, Tajik AJ, Reeder GS, Seward JB. Evaluation of hypertrophic cardiomyopathy by Doppler color flow imaging: initial observations. Mayo Clin Proc. 1986;61:631-9. 